Pacific Journal of Mathematics

THREE SPECTRAL THEOREMS FOR A PAIR OF SINGULAR 


\title{
THREE SPECTRAL THEOREMS FOR A PAIR OF SINGULAR FIRST-ORDER DIFFERENTIAL EQUATIONS
}

\author{
Bernard W. Roos and Ward C. SANGRen*
}

1. Preliminaries. In the regular case the classical method of obtaining eigenvalues and eigenfunctions of the equation

$$
y^{\prime \prime}(x)+[\lambda-q(x)] y=0 \quad\left[\prime \equiv \frac{d}{d x}\right]
$$

under Sturmian boundary conditions involves the use of asymptotic expansions. For the singular cases of (1) when the range of $x$ is infinite or semi-infinite instead of finite, Titchmarsh [6] has shown that such asymptotic solutions are also necessary in obtaining spectral and expansion theorems by the method of complex variables. The objective of this paper is to generalize for a particular case these types of results to the following pair of equations

$$
\begin{gathered}
u^{\prime}(x)-[\lambda a(x)+b(x)] v(x)=0, \\
v^{\prime}(x)+[\lambda c(x)+d(x)] u(x)=0 .
\end{gathered}
$$

Interest in this system arises from a consideration of the Dirac relativistic wave equations for a particle in a central field. The equations (2) correspond in this case to the radial wave equations. Conte and Sangren [2] and the authors [3] have shown that most of the results of Titchmarsh can be generalized for (2) over the interval $(0 \leqq x<\infty)$, under the restriction $a(x)=c(x)=1$. Also, the spectral properties of (2) for $a(x)=$ $c(x)=1$ over the infinite interval $(-\infty, \infty)$ have been investigated [4]. In this paper a discussion of the system (2) for $a(x)=x^{2 k}, c(x)=x^{-2 k}$ over the interval $(0, \infty)$ is presented. It is assumed throughout, that $k$ is a nonzero integer.

Let $\phi(x, \lambda)=\left[\phi_{1}(x, \lambda), \phi_{2}(x, \lambda)\right]$ and $\theta(x, \lambda)=\left[\theta_{1}(x, \lambda), \theta_{2}(x, \lambda)\right]$ be two solutions of system (2) over the interval $a \leqq x \leqq b$, where $a>0$ and $b<\infty$, such that $\phi_{1}(l, \lambda)=1, \phi_{2}(l, \lambda)=0, \theta_{1}(l, \lambda)=0, \theta_{2}(l, \lambda)=1$, where $a \leqq l \leqq b$. It can be shown that the Wronskian $W_{x}[\phi, \theta]=\phi_{1} \theta_{2}-\phi_{2} \theta_{1}$ is independent of $x$ so that since $W_{l}(\phi, \theta]=1, \phi(x, \lambda)$ and $\theta(x, \lambda)$ are linearly independent. For the singular case it can be shown that for complex values of $\lambda$ the system (2) has a solution $\psi(x, \lambda)=\left[\psi_{1}, \psi_{2}\right]=$ $\theta(x, \lambda)+m(\lambda) \phi(x, \lambda)$. A limit circle case is determined separately at each of the end points, 0 and $\infty$, by the conditions that all functions $c|\psi|^{2}$ $+a\left|\psi_{2}\right|^{2}$ are integrable, that is, belong to the class $L(0, l)$ or $L(l, \infty)$. In the limit point case, at either end, there exist only one $m(\lambda)$ and

Received January 6, 1961. Presented to the American Mathematical Society, Pasadena California, November 19, 1960.

* Present address: Computer Applications, Inc., San Diego, California. 
$\psi(x, \lambda)$ where $c\left|\psi_{1}\right|^{2}+a\left|\psi_{2}\right|^{2}$ is integrable. The existence at each end of at least one such integrable function is guaranteed by the direct extension of Weyl's limit-point, limit-circle theorem $[6,1,5]$. Furthermore, the $m(\lambda)$ at either end is such that $\overline{m(\lambda)}=m(\bar{\lambda})$ and $m(\lambda)$ is analytic in either the upper or lower half plane. The spectral properties can be obtained from these functions, $m_{0}(\lambda)$ and $m_{\infty}(\lambda)$.

2. The nature of the spectrum; interval $(1, \infty)$. To obtain the spectral properties of the system (2) over the interval $(1, \infty)$ the order properties as $x \rightarrow \infty$ of the solution vectors $\phi$ and $\theta$ have to be investigated. This is most easily done by using the corresponding integral equations which may be obtained by the method of variation of coefficients. It is easily verified that if $\phi_{1}(1)=1, \phi_{2}(1)=0, \theta_{1}(1)=0$ and $\theta_{2}(1)=1$ the appropriate integral equations are given by

$$
\begin{aligned}
& \phi_{1}(x, \lambda)=G_{1}(\lambda x)+\int_{1}^{x}\left\{b \phi_{2}\left[H_{2} G_{1}(\lambda x)-G_{2} H_{1}(\lambda x)\right]\right. \\
&\left.+d \phi_{1}\left[H_{1} G_{1}(\lambda x)-G_{1} H_{1}(\lambda x)\right]\right\} d s \\
&=G_{1}(\lambda x)+L\left(x, \phi_{1}, \phi_{2}\right) \\
& \phi_{2}(x, \lambda)=G_{2}(\lambda x)+\int_{1}^{x}\left\{b \phi_{2}\left[H_{2} G_{2}(\lambda x)-G_{2} H_{2}(\lambda x)\right]\right. \\
&\left.+d \phi_{1}\left[H_{1} G_{2}(\lambda x)-G_{1} H_{2}(\lambda x)\right]\right\} d s, \\
&= G_{2}(\lambda x)+K\left(x, \phi_{1}, \phi_{2}\right) \\
& \theta_{1}(x, \lambda)=H_{1}(\lambda x)+L\left(x, \theta_{1}, \theta_{2}\right) \\
& \theta_{2}(x, \lambda)=H_{2}(\lambda x)+K\left(x, \theta_{1}, \theta_{2}\right)
\end{aligned}
$$

where

$$
\begin{aligned}
G_{1}(\lambda x) & =+\frac{x^{p}}{\Delta}\left[J_{-(p-1)}(\lambda) J_{p}(\lambda x)+J_{p-1}(\lambda) J_{-p}(\lambda x)\right], \\
G_{2}(\lambda x) & =+\frac{x^{-(p-1)}}{\Delta}\left[J_{-(p-1)}(\lambda) J_{p-1}(\lambda x)-J_{p-1}(\lambda) J_{-(p-1)}(\lambda x)\right], \\
H_{1}(\lambda x) & =+\frac{x^{p}}{\Delta}\left[J_{-p}(\lambda) J_{p}(\lambda x)-J_{p}(\lambda) J_{-p}(\lambda x)\right] \\
H_{2}(\lambda x) & =+\frac{x^{-(p-1)}}{\Delta}\left[J_{-p}(\lambda) J_{p-1}(\lambda x)+J_{p}(\lambda) J_{-(p-1)}(\lambda x)\right] \\
\Delta & =J_{p}(\lambda) J_{-(p-1)}(\lambda)+J_{-p}(\lambda) J_{p-1}(\lambda)=\frac{2 \cos k \pi}{\pi \lambda}
\end{aligned}
$$

and

$$
p=k+\frac{1}{2}
$$


Let $\lambda=\sigma+i t$ where $t>0$, and let

$$
h_{1}(x, \lambda)=x^{-k} e^{-t x} \phi_{1}(x, \lambda) \text { and } h_{2}(x, \lambda)=x^{k} e^{-t x} \phi_{2}(x, \lambda) .
$$

The well-known asymptotic formula $J_{p}(z)=(2 / \pi z)^{1 / 2} \cos (z-1 / 2 p \pi-\pi / 4)$ $+0\left\{\left.\left|e^{-i z} /\right| z\right|^{-3 / 2}\right\}$ for $0 \leqq \arg z \leqq \pi / 2$ and $z \gg 1$ is used in the following. The substitution of $h_{1}$ and $h_{2}$ in (4) and (5) and the taking of absolute values yields

$$
\left|h_{1}(x, \lambda)\right| ;\left|h_{2}(x, \lambda)\right| \leqq M+\int_{1}^{x}\left\{\left|g_{1}\right|\left|h_{2}\right|+\left|g_{2}\right|\left|h_{1}\right|\right\} d s
$$

where

$$
\begin{gathered}
M=\max _{x \rightarrow \infty}\left\{\frac{x^{1 / 2}}{|\Delta|}\left[J_{p}(\lambda x) J_{-p+1}(\lambda)+J_{-p}(\lambda x) J_{p-1}(\lambda)\right] ;\right. \\
\left.\frac{x^{1 / 2}}{|\Delta|}\left[-J_{p-1}(\lambda x) J_{-p+1}(\lambda)+J_{-p+1}(\lambda x) J_{p-1}(\lambda)\right]\right\} \\
g_{1}=\max _{x \rightarrow \infty}\left\{\left[J_{p}(\lambda x) J_{-p+1}(\lambda s)+J_{-p}(\lambda x) J_{p-1}(\lambda s)\right] ;\right. \\
\left.\left[J_{p-1}(\lambda x) J_{-p+1}(\lambda s)-J_{-p+1}(\lambda x) J_{p-1}(\lambda s)\right]\right\} \\
\left\{\frac{x^{1 / 2}}{|\Delta|} e^{-t(x-s)} b(s) s^{-2 k+1 / 2}\right\} \\
g_{2}=\max _{x \rightarrow \infty}\left\{\left[J_{-p}(\lambda x) J_{p}(\lambda s)-J_{p}(\lambda x) J_{-p}(\lambda s)\right] ;\right. \\
\left.\left[J_{-p+1}(\lambda x) J_{p}(\lambda s)+J_{p-1}(\lambda x) J_{-p}(\lambda s)\right]\right\} \\
\left\{\frac{x^{1 / 2}}{|\Delta|} e^{-t(x-s)} d(s) s^{2 k+1 / 2}\right\} .
\end{gathered}
$$

According to the lemma in [3] this can be reduced to the inequality

$$
h_{1}(x, \lambda) ; h_{2}(x, \lambda) \leqq M \exp \left\{\int_{1}^{x}\left(g_{1}+g_{2}\right) d s\right\} \text {. }
$$

Consequently, it can be concluded that when $b(x) x^{-2 k}$ and $d(x) x^{2 \pi}$ are both $L(1, \infty), h_{1}(x, \lambda)$ and $h_{2}(x, \lambda)$ will both be bounded for all values of $x$. Moreover, for large $x$

$$
\phi_{1}(x, \lambda)=0\left\{e^{t x} x^{k}\right\}, \phi_{2}(x, \lambda)=0\left\{e^{t x} x^{-k}\right\} .
$$

Furthermore, when $\operatorname{Im} \lambda=0$, that is for real $\lambda$, and $x \rightarrow \infty$ the order properties for $\phi$ and $\theta$ may be written as follows:

$$
\begin{aligned}
& \phi_{1}(x, \lambda)=x^{p}\left\{\mu(\lambda) J_{p}(\lambda x)+\nu(\lambda) J_{-p}(\lambda x)+o(1)\right\} \\
& \phi_{2}(x, \lambda)=x^{-p+1}\left\{+\mu(\lambda) J_{p-1}(\lambda x)-\nu(\lambda) J_{-p+1}(\lambda x)+o(1)\right\} \\
& \theta_{1}(x, \lambda)=x^{p}\left\{\xi(\lambda) J_{p}(\lambda x)+\eta(\lambda) J_{-p}(\lambda x)+o(1)\right\} \\
& \theta_{2}(x, \lambda)=x^{-p+1}\left\{\xi(\lambda) J_{p-1}(\lambda x)-\eta(\lambda) J_{-p+1}(\lambda x)+o(1)\right\}
\end{aligned}
$$

where 
(7)

$$
\begin{aligned}
& \mu(\lambda)=\frac{1}{\Delta}\left[J_{-p+1}(\lambda)-\int_{1}^{\infty}\left[-b(s) \phi_{2}(s) s^{-p+1} J_{-p+1}(\lambda s)+d(s) \phi_{1}(s) s^{p} J_{-p}(\lambda s)\right] d s\right] \\
& \nu(\lambda)=\frac{1}{\Delta}\left[J_{p-1}(\lambda)-\int_{1}^{\infty}\left[-b(s) \phi_{2}(s) s^{-p+1} J_{p-1}(\lambda s)+d(s) \phi_{1}(s) s^{p} J_{p}(\lambda s)\right] d s\right] \\
& \xi(\lambda)=\frac{1}{\Delta}\left[+J_{-p}(\lambda)-\int_{1}^{\infty}\left[-b(s) \theta_{2}(s) s^{-p+1} J_{-p+1}(\lambda s)+d(s) \theta_{1}(s) s^{p} J_{-p}(\lambda s)\right] d s\right] \\
& \eta(\lambda)=\frac{1}{\Delta}\left[-J_{p}(\lambda)-\int_{1}^{\infty}\left[-b(s) \theta_{2}(s) s^{-p+1} J_{p-1}(\lambda s)+d(s) \theta_{1}(s) s^{p} J_{-p}(\lambda s)\right] d s\right] .
\end{aligned}
$$

The integrals in (7) converge uniformly in $\lambda$ hence, $\mu(\lambda), \nu(\lambda), \xi(\lambda), \eta(\lambda)$ will be continuous and bounded functions of $\lambda$.

When $\lambda$ is complex and has an imaginary part greater than zero, Im $\lambda>0$, and when $b(x) x^{-2 \pi}$ and $d(x) x^{2 k}$ are both $L(1, \infty)$, one can obtain

where

$$
\begin{aligned}
& \phi_{1}(x, \lambda)=x^{k} e^{-i \lambda x}\left[M_{1}(\lambda)+o(1)\right] \\
& \phi_{2}(x, \lambda)=x^{-k} e^{-i \lambda x}\left[M_{2}(\lambda)+o(1)\right] \\
& \theta_{1}(x, \lambda)=x^{k} e^{-i \lambda x}\left[N_{1}(\lambda)+o(1)\right] \\
& \theta_{2}(x, \lambda)=x^{-k} e^{-i \lambda x}\left[N_{2}(\lambda)+o(1)\right]
\end{aligned}
$$

$$
\begin{aligned}
M_{1}(\lambda)= & \frac{(2 \pi \lambda)^{-1 / 2} e^{i k \pi / 2}}{\Delta}\left\{i J_{-p+1}(\lambda)+i J_{p-1}(\lambda) \cos k \pi\right. \\
& +\int_{1}^{\infty} b(s) \phi_{2}(s) s^{-p+1}\left[i J_{-p+1}(\lambda s)+J_{p-1}(\lambda s) \cos k \pi\right] d s \\
& \left.+\int_{1}^{\infty} d(s) \phi_{1}(s) s^{p}\left[J_{p}(\lambda s) \cos k \pi-J_{-p}(\lambda s)\right] d s\right\} \\
M_{2}(\lambda)= & \frac{(2 \pi \lambda)^{-1 / 2} e^{i k \pi / 2}}{\Delta}\left\{+J_{-p+1}(\lambda)-i J_{p-1}(\lambda) \cos k \pi\right. \\
& +\int_{1}^{\infty} b(s) \phi_{2}(s) s^{-p+1}\left[J_{-p+1}(\lambda s)-i J_{p-1}(\lambda s) \cos k \pi\right] d s \\
& \left.-\int_{1}^{\infty} d(s) \phi_{1}(s) s^{p}\left[i J_{p}(\lambda s) \cos k \pi+J_{-p}(\lambda s)\right] d s\right\} \\
N_{1}(\lambda)= & \frac{(2 \pi \lambda)^{-1 / 2} e^{i k \pi / 2}}{\Delta}\left\{-i J_{-p}(\lambda)+J_{p}(\lambda) \cos k \pi\right. \\
& +\int_{1}^{\infty} b(s) \theta_{2}(s) s^{-p+1}\left[i J_{-p+1}(\lambda s)+J_{p-1}(\lambda s) \cos k \pi\right] d s \\
& \left.+\int_{1}^{\infty} d(s) \theta_{1}(s) s^{p}\left[J_{p}(\lambda s) \cos k \pi-i J_{-p}(\lambda s)\right] d s\right\} \\
N_{2}(\lambda)= & \frac{(2 \pi \lambda)^{-1 / 2} e^{i k \pi / 2}}{\Delta}\left\{J_{-p}(\lambda)+i J_{p}(\lambda) \cos k \pi\right. \\
& +\int_{1}^{\infty} b(s) \theta_{2}(s) s^{-p+1}\left[J_{-p+1}(\lambda s)-i J_{p-1}(\lambda s) \cos k \pi\right] d s \\
& \left.-\int_{1}^{\infty} d(s) \theta_{1}(s) s^{p}\left[i J_{p}(\lambda s) \cos k \pi+J_{-p}(\lambda s)\right] d s\right\} .
\end{aligned}
$$


Let $\psi(x, \lambda)=\theta(x, \lambda)+m(\lambda) \phi(x, \lambda)$ be that solution of (2) mentioned in the Preliminaries where $c\left|\psi_{1}\right|^{2}+a\left|\psi_{-2}\right|^{2}$ is $(1, \infty)$. $\psi$ is given by

$$
\begin{gathered}
\psi_{1}(x, \lambda)=\theta_{1}(x, \lambda)+m(\lambda) \phi_{1}(x, \lambda)=x^{k} e^{i \lambda x}\left[N_{1}(\lambda)+m(\lambda) M_{1}(\lambda)+o(1)\right], \\
\psi_{2}(x, \lambda)=\theta_{2}(x, \lambda)+m(\lambda) \phi_{2}(x, \lambda)=x^{-k} e^{-i \lambda x}\left[N_{2}(\lambda)+m(\lambda) M_{2}(\lambda)+o(1)\right] .
\end{gathered}
$$

It is apparent that in order for $c\left|\psi_{1}\right|^{2}+a\left|\psi_{2}\right|^{2}$ to be in $L(1, \infty)$ we must have

$$
m(\lambda)=-\frac{N_{1}(\lambda)}{M_{1}(\lambda)}=-\frac{N_{2}(\lambda)}{M_{2}(\lambda)}
$$

When $\operatorname{Im} \lambda \rightarrow 0$, it follows from (7) and (9)that

$$
\begin{aligned}
& M_{1}(\lambda) \rightarrow(2 \pi \sigma)^{-1 / 2} e^{i k \pi / 2}[i \mu(\sigma)+\nu(\sigma) \cos k \pi] \\
& M_{2}(\lambda) \rightarrow(2 \pi \sigma)^{-1 / 2} e^{i k \pi / 2}[+\mu(\sigma)-i \nu(\sigma) \cos k \pi] \\
& N_{1}(\lambda) \rightarrow(2 \pi \sigma)^{-1 / 2} e^{i k \pi / 2}[i \xi(\sigma)+\eta(\sigma) \cos k \pi] \\
& N_{2}(\lambda) \rightarrow(2 \pi \sigma)^{-1 / 2} e^{i k \pi / 2}[+\xi(\sigma)-i \eta(\sigma) \cos k \pi] .
\end{aligned}
$$

Hence,

$$
\begin{gathered}
\lim _{t \rightarrow 0} m(\lambda)=-\frac{i \xi(\sigma)+\eta(\sigma) \cos k \pi}{i \mu(\sigma)+\nu(\sigma) \cos k \pi} \\
=-\cos k \pi \frac{\mu \eta-\nu \xi}{\nu^{2}(\sigma)+\mu^{2}(\sigma)} .
\end{gathered}
$$

From (6) one also obtains

$$
W_{x}[\phi, \theta]=\phi_{1} \theta_{2}-\phi_{2} \theta_{1}=\Delta[\nu \xi-\mu \eta]+o(1) .
$$

Because $W_{0}[\phi, \theta]=1$,

$$
\nu \xi-\mu \eta=\frac{1}{\Delta}
$$

The substitution of this result in (12) yields

$$
\lim _{t \rightarrow 0} \operatorname{Im} m(\lambda)=-\frac{\sigma \pi}{\nu^{2}(\sigma)+\mu^{2}(\sigma)} .
$$

Hence, $\operatorname{Im} m(\lambda)$ is a nonpositive, nonvanishing continuous and bounded function of $\lambda$ for both positive and negative $\lambda$.

This is easily seen for positive $\lambda$ since $\lambda, \nu^{2}$ and $\mu^{2}$ are all positive numbers. For negative $\lambda$ not only is $\lambda$ negative, but so are $\nu^{2}$ and $\mu^{2}$. This can be verified by making use of the formula

$$
J_{p}\left(i^{2 m} z\right)=i^{2 m p} J_{p}(z) \text {. }
$$

The spectrum is, therefore, continuous over the infinite interval $-\infty<\lambda<\infty$. These results may be summarized in the following theorem, 
THEOREM 1. Consider the system (2) where $a(x)=x^{2 k}$ and $c(x)=x^{-2 k}$ over the semi-infinite interval $[l, \infty]$ and under the boundary conditions

$$
u(l) \cos \alpha+v(l) \sin \alpha=0
$$

Let $b(x)$ and $d(x)$ be real-valued continuous functions of $x$ and let $b(x) x^{-2 k}$ and $d(x) x^{2 k}$ belong to the class $L(l, \infty)$. A solution of the system (2), (13) is defined as a vector function $[u(x, \lambda), v(x, \lambda)]$ with continuous first derivatives satisfying this system. The values of $\lambda$ for which such solutions exists form a continuous spectrum over the real $\lambda$-axis $(-\infty<\lambda<\infty)$.

In order to simplify the appearance of the equations in the preceeding proof and in the following, it was assumed, without loss of generality, that $\alpha=\pi / 2$ and $l=1$.

\section{Nature of the spectrum; interval $(0,1)$.}

THEOREM 2. Consider the system (2) where $a(x)=x^{2 k}, c(x)=x^{-2 k}$ over the interval $0 \leqq x \leqq 1$ and subject to a linear homogeneous boundary condition at $x=1$. The spectrum is discrete provided:

$$
\begin{aligned}
& b(x) x^{-2(p-1)} \text { and } d(x) \text { are } L(0,1) \quad \text { for } p=k+1 / 2>1 \\
& b(x) \quad \text { and } d(x) \text { are } L(0,1) \quad \text { for } 0<p<1 \\
& b(x) \text { and } d(x) x^{2 p} \text { are } L(0,1) \text { for } p<0 \text {. }
\end{aligned}
$$

The proof of this theorem follows closely that of Theorem 1. Except for an obvious change of integration limits, the integral equations corresponding to system (2) of Theorem 2, are given by equations (4) and (5).

First, consider the case $p>0$. For $x \rightarrow 0$ one has the well-known asymptotic relations:

$$
J_{p}(\lambda x) \rightarrow \frac{(\lambda x)^{p}}{\Gamma(p+1) 2^{p}} \quad J_{-p}(\lambda x) \rightarrow \frac{(-1)^{k} \Gamma(p) 2^{p}(\lambda x)^{-p}}{\pi}
$$

and for $p-1>0$ :

$$
J_{p-1}(\lambda x) \rightarrow \frac{(\lambda x)^{p-1}}{\Gamma(p) 2^{p-1}} \quad J_{-p+1}(\lambda x) \rightarrow \frac{(-1)^{k-1} \Gamma(p-1)(\lambda x)^{-p+1} 2^{p-1}}{\pi} .
$$

Let $h_{1}(x, \lambda)=\phi_{1}(x, \lambda), h_{2}(x, \lambda)=x^{+2(p-1)} \phi_{2}(x, \lambda)$ and $\lambda=\sigma+i t$ where $t>0$. One obtains:

$$
\begin{aligned}
h_{1}(x, \lambda)= & \frac{x^{p}}{\Delta}\left[J_{-p+1}(x) J_{p}(\lambda x)+J_{p-1}(\lambda) J_{-p}(\lambda x)\right. \\
& -\int_{x}^{1}\left\{b s^{-3(p-1)} h_{2}(s, \lambda)\left[J_{-p}(x) J_{-p+1}(s)+J_{-p}(x) J_{p-1}(s)\right]\right. \\
& \left.\left.+d h_{1} s^{p}\left[J_{p}(\lambda s) J_{-p}(\lambda x) J_{-p}(\lambda x) J_{-p}(\lambda s)\right]\right\} d s\right]
\end{aligned}
$$




$$
\begin{aligned}
h_{2}(x, \lambda)= & \frac{x^{+(p-1)}}{\Delta}\left[J_{-p+1}(\lambda) J_{p-1}(\lambda x)-J_{p-1}(\lambda) J_{-p+1}(\lambda x)\right. \\
& -\int_{x}^{1}\left\{d s^{-3(p-1)} h_{2}(s)\left[J_{p-1}(x) J_{-p+1}(s)-J_{p-1}(s) J_{-p+1}(x)\right]\right. \\
& \left.\left.-d h_{1} s^{p}\left[J_{p}(\lambda s) J_{-p+1}(\lambda x)+J_{-p}(\lambda s) J_{p-1}(\lambda x)\right]\right\} d s\right] .
\end{aligned}
$$

For $x \rightarrow 0 h_{1}(x, \lambda)$ and $h_{2}(x, \lambda)$ are bounded for all $x$ provided $b x^{-2(p-1)}$ and $d$ are $L(0,1)$ (see lemma [3]). Hence, $\phi_{1}(x, \lambda)=o(1)$ and $\phi_{2}(x, \lambda)=o\left(x^{-2(p-1)}\right)$ for $x \rightarrow 0$.

It follows that one may write

$$
\begin{aligned}
& \phi_{1}(x, \lambda)=\left[M_{1}(\lambda)+o(1)\right], \\
& \phi_{2}(x, \lambda)=x^{-2(p-1)}\left[M_{2}(\lambda)+o(1)\right],
\end{aligned}
$$

where

$$
M_{1}(\lambda)=\frac{(-1)^{k} \Gamma(p) 2^{p} \lambda^{-p}}{\pi \Delta}\left[J_{p-1}(\lambda)-\int_{0}^{1}\left[b \phi_{2} J_{p-1}(\lambda s) s^{-p+1}+d \phi_{1} J_{p}(\lambda s) s^{p}\right] d s\right]
$$

and

$$
\begin{aligned}
M_{2}(\lambda)= & \frac{(-1)^{k-1} \Gamma(p-1) 2^{p-1} \lambda^{-p-1}}{\pi \Delta} \\
& {\left[-J_{p-1}(\lambda)+\int_{0}^{1}\left[J_{p-1}(\lambda s) b \phi_{2} s^{-p+1}-d \phi_{1} J_{p}(\lambda s) s^{p}\right] d s\right] . }
\end{aligned}
$$

Similarly,

$$
\begin{aligned}
& \theta_{1}(x, \lambda)=\left[N_{1}(\lambda)+o(1),\right. \\
& \theta_{2}(x, \lambda)=x^{-2(p-1)}\left[N_{2}(\lambda)+o(1)\right],
\end{aligned}
$$

where

$$
N_{1}(\lambda)=\frac{(-1)^{k} \Gamma(p) 2^{p} \lambda^{-p}}{\pi \Delta}\left[+J_{+p}(\lambda)-\int_{0}^{1}\left\{b \theta_{2} s^{-p+1} J_{p-1}(\lambda s)+d \theta_{1} s^{p} J_{p}(\lambda s)\right\} d s\right]
$$

and

$$
\begin{aligned}
N_{2}(\lambda)= & \frac{(-1)^{k-1} \Gamma(p-1) 2^{p-1} \lambda^{-(p-1)}}{\pi \Delta} \\
& {\left[-J_{p}(\lambda)+\int_{0}^{1}\left\{b \theta_{2} s^{-p+1} J_{p-1}(\lambda s)-d \theta_{1} s^{p} J_{p}(\lambda s)\right\} d s\right] . }
\end{aligned}
$$

A similar argument as in $\S 3$, yields

$$
m(\lambda)=-\frac{N_{1}(\lambda)}{M_{1}(\lambda)}=-\frac{N_{2}(\lambda)}{M_{2}(\lambda)}
$$




$$
m(\lambda)=\frac{J_{p}(\lambda)-\int_{0}^{1}\left\{b \theta_{2} s^{-p+1} J_{p-1}(\lambda s)+d \theta_{1} s^{p} J_{p}(\lambda s)\right\} d s}{J_{p-1}(\lambda)-\int_{0}^{1}\left\{b \theta_{2} s^{-p+1} J_{p-1}(\lambda s)+d \phi_{1} s^{p} J_{p}(\lambda s)\right\} d s}
$$

For $t \rightarrow 0$, i.e., $\operatorname{Im} \lambda \rightarrow 0, M_{1}(\lambda)$ and $N_{1}(\lambda)$ tend to real limits. This is apparent for $\lambda>0$ and is easily shown for $\lambda<0$ by using again the relation $J_{r}\left(z e^{i m \pi}\right)=e^{i m \pi r} J_{r}(z)$. Hence, $\operatorname{Im} m(\lambda) \rightarrow 0$ except possibly at the zeros of $M_{1}(\lambda)$. Consequently, the spectrum will be discrete.

For values of the parameter $p$ such that $0<p<1$ one has

$$
\begin{gathered}
J_{p}(\lambda x) \rightarrow \frac{(\lambda x)^{p}}{\Gamma(p+1) 2^{p}}, \quad J_{-p}(\lambda x) \rightarrow \frac{(-1)^{k} \Gamma(p) 2^{p}(\lambda x)^{-p}}{\pi}, \\
J_{p-1}(\lambda x) \rightarrow \frac{(-1)^{k-1} \Gamma(p-1)(\lambda x)^{(p-1)} 2^{p-1}}{\pi}, \quad J_{-p+1}(\lambda x) \rightarrow \frac{(\lambda x)^{-p+1}}{\Gamma(p) 2^{p-1}} .
\end{gathered}
$$

It is not difficult to verify, applying the same method as discussed above, that in this case, when $b(x)$ and $d(x)$ are $L(0,1)$ the limit circle case prevails and the spectrum is discrete. $k$, an integer, is not in this interval.

For $p<0$ the asymptotic expressions become

$$
\begin{gathered}
J_{p}(\lambda x) \rightarrow \frac{(-1)^{k} \Gamma(p) 2^{p}(\lambda x)^{+p}}{\pi}, \quad J_{-p}(\lambda x) \rightarrow \frac{(\lambda x)^{-p}}{\Gamma(p+1) 2^{p}}, \\
J_{p-1}(\lambda x) \rightarrow \frac{(-1)^{k-1} \Gamma(p-1) 2^{p-1}(\lambda x)^{+(p-1)}}{\pi}, \quad J_{-(p-1)}(\lambda x) \rightarrow \frac{(\lambda x)^{-(p-1)}}{\Gamma(p) 2^{p-1}} .
\end{gathered}
$$

In a similar fashion, for the case where $p>1$ one can obtain that $\phi_{1}(x, \lambda)=o\left(x^{2 p}\right)$ and $\phi_{2}(x, \lambda)=o(1)$. In this situation the additional condition that $b$ and $d(x) x^{2 p}$ are in $L(0,1)$ must be imposed. The conclusion that the spectrum will be discrete follows accordingly.

4. Nature of the spectrum; interval $(0, \infty)$.

In this case singularities exist at both ends of the interval and it has been shown [4] that the spectral properties of the system (2) are determined by the imaginary parts of the following functions:

$$
\frac{1}{m_{0}-m_{\infty}}, \frac{m_{0}}{m_{0}-m_{\infty}}, \frac{m_{0} m_{\infty}}{m_{0}-m_{\infty}},
$$

where $m_{0}(\lambda)$ and $m_{\infty}(\lambda)$ are the previously determined $m$ 's at $x=0$ and $\infty$. As was shown in the previous sections $m_{0}(\lambda)$ is a meromorphic function while $m_{\infty}$ has a nonvanishing imaginary part. It is clear that the imaginary parts of the three functions above tend to finite limits which can vanish at most at discrete points. The associated spectrum will therefore be continuous over the whole real $\lambda$-axis.

THEOREM 3. Consider the system (2) where $a(x)=x^{2 k}$ and $c(x)=x^{-2 k}$ 
over the interval $0 \leqq x \infty$. The spectrum will be continuous over the entire real $\lambda$-axis $(-\infty<\lambda<\infty)$, provided $b(x)$ and $d(x)$ satisfy the conditions given in Theorem 1 and Theorem 2.

\section{REFERENCES}

1. E. A. Coddington and N. Levinson, Theory of ordinary differential equations, Mac Graw-Hill New York, 1955.

2. S. D. Conte, and W. C. Sangren, An Expansion theorem for a pair of first order equations, Can. J. Math., 6 (1954), 554-560.

3. B. W. Roos and W. C. Sangren, Spectra for a pair of singular first order differential equations, Proc. Amer. Math. Soc., 12 (1961), 468-476.

4. —, General Atomic Report (unpublished).

5. - General Atomic Report GA-1777.

6. E. C. Titchmarsh, Eigenfunction expansions associated with second-order differential equations, Oxford, 1946.

General Dynamics CorP.

John Jay Hopkins Laboratory For PuRe and Applied Science

GeNeral ATOMIC Division

GENERAL DYNAMICS CORPORATION 



\title{
PACIFIC JOURNAL OF MATHEMATICS
}

\author{
EDITORS
}

RalPh S. Phillips

Stanford University

Stanford, California

M. G. Arsove

University of Washington

Seattle 5 , Washington
A. L. Whiteman

University of Southern California Los Angeles 7, California

LOWell J. PAIGE

Unıversity of California

Los Angeles 24, California

\section{ASSOCIATE EDITORS}
E. F. BECKENBACH
D. DERRY
H. L. ROYDEN
E. G. STRAUS
T. M. CHERRY
M. OHTSUKA
E. SPANIER
F. WOLF

\section{SUPPORTING INSTITUTIONS}

\author{
UNIVERSITY OF BRITISH COLUMBIA \\ CALIFORNIA INSTITUTE OF TECHNOLOGY \\ UNIVERSITY OF CALIFORNIA \\ MONTANA STATE UNIVERSITY \\ UNIVERSITY OF NEVADA \\ NEW MEXICO STATE UNIVERSITY \\ OREGON STATE UNIVERSITY \\ UNIVERSITY OF OREGON \\ OSAKA UNIVERSITY \\ UNIVERSITY OF SOUTHERN CALIFORNIA
}

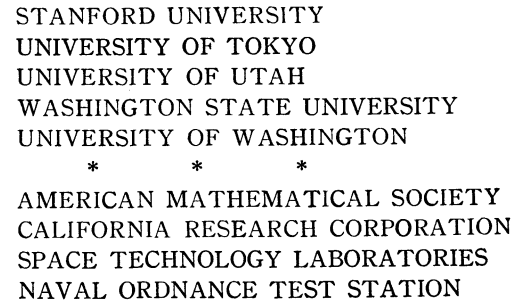

Mathematical papers intended for publication in the Pacific Journal of Mathematıcs should be typewritten (double spaced), and the author should keep a complete copy. Manuscripts may be sent to any one of the four editors. All other communications to the editors should be addressed to the managing editor, L. J. Paige at the University of California, Los Angeles 24, California.

50 reprints per author of each article are furnıshed free of charge; additional copies may be obtained at cost in multiples of 50 .

The Pacific Journal of Mathematics is published quarterly, in March, June, September, and December. Effective with Volume 13 the price per volume (4 numbers) is $\$ 18.00$; single issues, $\$ 5.00$. Special price for current issues to individual faculty members of supporting institutions and to individual members of the American Mathematical Society: $\$ 8.00$ per volume; single issues $\$ 2.50$. Back numbers are available.

Subscriptions, orders for back numbers, and changes of address should be sent to Pacific Journal of Mathematics, 103 Highland Boulevard, Berkeley 8, California.

Printed at Kokusai Bunken Insatsusha (International Academic Printing Co., Ltd.), No. 6 , 2-chome, Fujimi-cho, Chiyoda-ku, Tokyo, Japan.

PUBLISHED BY PACIFIC JOURNAL OF MATHEMATICS, A NON-PROFIT CORPORATION

The Supporting Institutions listed above contribute to the cost of publication of this Journal, but they are not owners or publishers and have no responsibility for its content or policies. 


\section{Pacific Journal of Mathematics}

\section{Vol. 12, No. $3 \quad$ March, 1962}

Alfred Aeppli, Some exact sequences in cohomology theory for Kähler

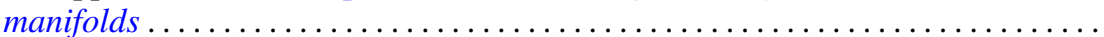

Paul Richard Beesack, On the Green's function of an N-point boundary value

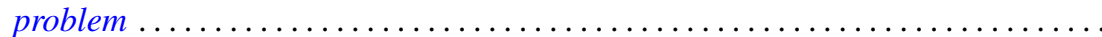

James Robert Boen, On p-automorphic p-groups....

James Robert Boen, Oscar S. Rothaus and John Griggs Thompson, Further results

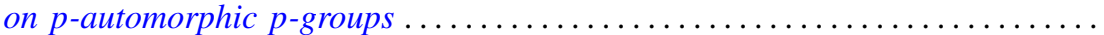

James Henry Bramble and Lawrence Edward Payne, Bounds in the Neumann problem for second order uniformly elliptic operators ..................

Chen Chung Chang and H. Jerome (Howard) Keisler, Applications of ultraproducts of pairs of cardinals to the theory of models .........................

Stephen Urban Chase, On direct sums and products of modules ................

Paul Civin, Annihilators in the second conjugate algebra of a group algebra .......

J. H. Curtiss, Polynomial interpolation in points equidistributed on the unit

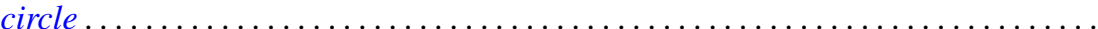

Marion K. Fort, Jr., Homogeneity of infinite products of manifolds with

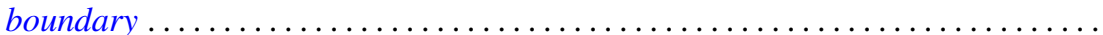

James G. Glimm, Families of induced representations . . . . . . . . . . . . . . .

Daniel E. Gorenstein, Reuben Sandler and William H. Mills, On almost-commuting

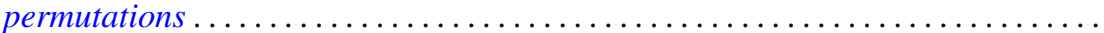

Vincent C. Harris and M. V. Subba Rao, Congruence properties of $\sigma_{r}(N) \ldots \ldots \ldots$

Harry Hochstadt, Fourier series with linearly dependent coefficients . . . . . . . . . . 925

Kenneth Myron Hoffman and John Wermer, A characterization of $C(X)$. .

Robert Weldon Hunt, The behavior of solutions of ordinary, self-adjoint differential equations of arbitrary even order...

Edward Takashi Kobayashi, A remark on the Nijenhuis tensor

David London, On the zeros of the solutions of $w^{\prime \prime}(z)+p(z) w(z)=0$

Gerald R. Mac Lane and Frank Beall Ryan, On the radial limits of Blaschke products...

T. M. MacRobert, Evaluation of an E-function when three of its upper parameters

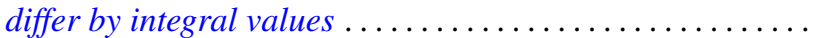

Robert W. McKelvey, The spectra of minimal self-adjoint extensions of a symmetric operator

Adegoke Olubummo, Operators of finite rank in a reflexive Banach space. .

David Alexander Pope, On the approximation of function spaces in the calculus of variations

Bernard W. Roos and Ward C. Sangren, Three spectral theorems for a pair of singular first-order differential equations...............

Arthur Argyle Sagle, Simple Malcev algebras over fields of characteristic zero .

S. C. Tang, Some theorems on the ratio of empirical distribution to the theoretical distribution

Robert Charles Thompson, Normal matrices and the normal basis in abelian number fields.

Howard Gregory Tucker, Absolute continuity of infinitely divisible distributions ... 